Address for Correspondence: Dr. Bushra Siddiqui,

Department of Pathology, JN Medical College, Aligarh Muslim University,

Aligarh, Uttar Pradesh - 202 002, India.

E-mail: bushrasiddiqui85@yahoo.com

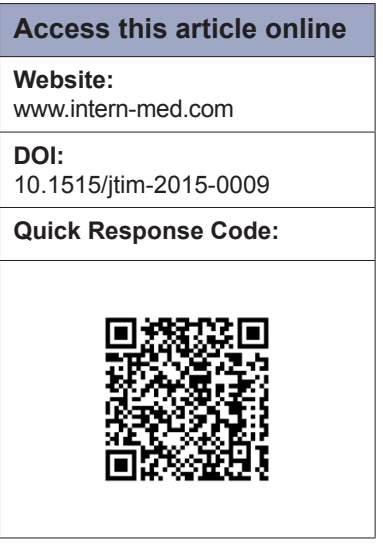

\title{
Primary hydatid disease of the breast clinically masquerading as a galactocele: A case report
}

\author{
Bushra Siddiqui, Shahbaz Habib Faridi ${ }^{1}$, Sayeedul Hasan Arif, \\ Mohammad Aslam ${ }^{1}$ \\ Departments of Pathology and 'Surgery, JN Medical College, Aligarh Muslim University, Aligarh, \\ Uttar Pradesh, India
}

\section{ABSTRACT}

We report a rare case of a 30-year-old breastfeeding woman who presented to our surgical outpatient department with complaints of a gradually enlarging lump in her left breast for the last 2 months. She also complained of difficulty in breastfeeding for 1 week. On examination, the right breast was normal but there was a $4 \mathrm{~cm} \times 4 \mathrm{~cm}$ retroareolar soft lump in her left breast. Examination of the bilateral axilla was normal. A provisional diagnosis of galactocele was made and the patient was subjected to ultrasonic mammography and fine needle aspiration cytology (FNAC). Ultrasonic mammography of the left breast showed a heterogeneous, hypoechoic lesion with thick septations and internal echoes suggestive of a complex cystic lesion, leading to a differential diagnosis of either hydatid cyst or breast abscess. On FNAC, the aspirate was not milky and cytological examination was performed, which revealed hydatid scolices, hooklets, foamy macrophages and granular debris, leading to the final diagnosis of hydatid cyst of the breast. The patient was investigated further but there were no cysts at any other site. Chemotherapy with albendazole was started and surgery was performed after 1 month. Histopathology further confirmed the diagnosis. Owing to the rarity of presentation, this case is being reported here.

Key words: Fine needle aspiration, hydatid cyst, ultrasonic mammography

\section{INTRODUCTION}

Hydatid cyst disease is a zoonotic infection that results from tissue infestation with the larval stage of the parasite Ecbinococcus granulosus. ${ }^{[1]}$ The definitive hosts of the parasite are dogs, whereas the intermediate hosts are sheep and other ruminants. Humans are accidental intermediate hosts of this organism. It is an endemic disease that particularly affects people who live in rural areas in intimate contact with cattle. The primary location is mostly in the liver (75\%) and lungs (15\%), with only $10 \%$ occurring in other parts of the body. ${ }^{[2]}$ Primary hydatid disease of the breast is extremely rare even in endemic areas, accounting for $0.27 \%$ of all cases. ${ }^{[3,4]}$ Hydatid cyst of the breast is rarely kept as a differential diagnosis of breast lump owing to the rarity of the disease. Preoperative diagnosis of the condition is also very difficult. ${ }^{[5]}$ We describe a rare case of isolated hydatid cyst of the breast in a breastfeeding woman in whom a preoperative diagnosis was made on cytological examination of the cyst fluid.

\section{CASE REPORT}

A 30-year-old breastfeeding female patient presented to our surgical outpatient department with complaints of a painless lump along with decreased milk secretion from her left breast. On examination, the right breast was normal but there was a $4 \mathrm{~cm} \times 4 \mathrm{~cm}$ well-defined soft lump present in the retroareolar region of her left breast. The temperature of the swelling was not raised. Examination of the axilla was normal. A provisional diagnosis of galactocele was made. On ultrasonic mammography, heterogeneous predominantly hypoechoic lesions in 
the central quadrant of the left breast along with few anechoic cysts at the peripheral aspect of the lesion were seen, suggestive of hydatid cyst or breast abscess [Figure 1].

Fine needle aspiration cytology (FNAC) of the lump was performed, which yielded creamish white nonmilky fluid. Microscopic examination of hematoxylin and eosin-stained smears showed hydatid scolices, hooklets foamy macrophages and granular debris consistent with the diagnosis of hydatid cyst of the breast [Figure 2a and b].

The patient was further investigated by ultrasonography (USG) of the abdomen and chest radiograph, which were normal. Preoperative chemotherapy with albendazole was started at a dose of $10 \mathrm{mg} / \mathrm{kg} /$ day for 1 month, after which the size of the cyst decreased.

The patient was operated after 1 month. Aspiration of the cyst was performed after injecting 20\% saline and the surrounding tissue was packed with mops soaked in 3\% saline. Complete surgical resection of the cystic mass from the surrounding breast tissue was performed but the cyst ruptured accidentally; however, there was no anaphylactic reaction [Figure 3]. She was discharged in a satisfactory condition on the fifth postoperative day on albendazole therapy for another 28 days to prevent recurrence. Owing to the rarity of the site and such rare clinical presentation of hydatid disease, this case is being reported here.

\section{DISCUSSION}

Hydatid disease is a parasitic infection caused by Echinococcus, mostly by Echinococcus granulosus. Hydatid disease of the breast is a very rare entity $(0.27 \%)$ even in endemic areas; it can be the only primary site or part of disseminated hydatidosis. ${ }^{[3,4]}$

Patients usually present with a painless breast lump that increases in size over time. It generally affects women between 30 and 50 years of age, although ages from 20 to 74 years have also been reported. ${ }^{[6]}$ It should be differentiated from fibroadenoma in young patients and carcinoma in older patients. ${ }^{[7]}$ When secondary infection occurs, hydatid cyst of the breast cannot be distinguished from breast abscess, clinically or by mammography. ${ }^{[8]}$ Triple assessment, i.e. clinical assessment, USG and FNAC, is used for the diagnosis of breast lumps. In the present case, clinical assessment was suggestive of galactocele but FNAC and USG were diagnostic. Serological tests such as enzyme-linked immunosorbent assay for Echinococcus can also be used for the preoperative diagnosis of hydatid

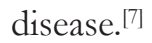

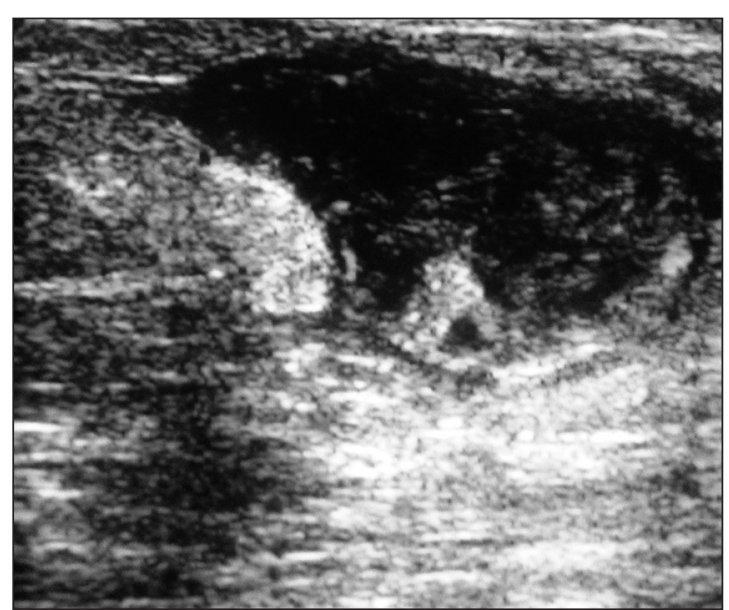

Figure 1: Ultrasonic mammography image of the left breast showing heterogeneous, hypoechoic lesion with thick septations and internal echoes suggestive of complex cystic lesion

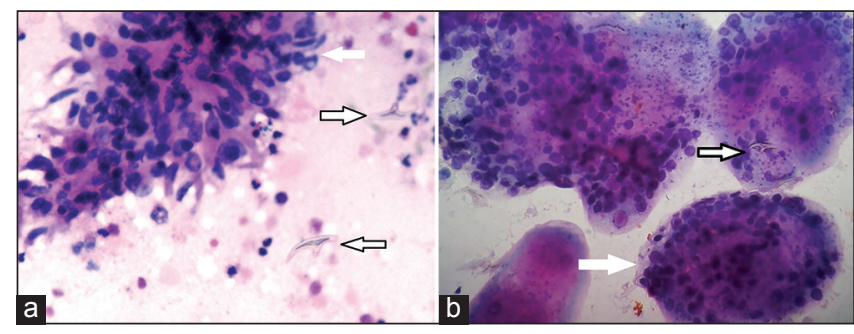

Figure 2: (a) Smear from the cyst aspirate showing a cluster of benign breast duct epithelial cells and myoepithelial cells along with macrophages (white arrow), inflammatory cells and hydatid hooklets (black arrow) in the background, Hematoxylin and Eosin, $\times 400$. (b) High-power view showing multiple hydatid scolices (white arrow) with hooklets (black arrow), Hematoxylin and Eosin, $\times 400$

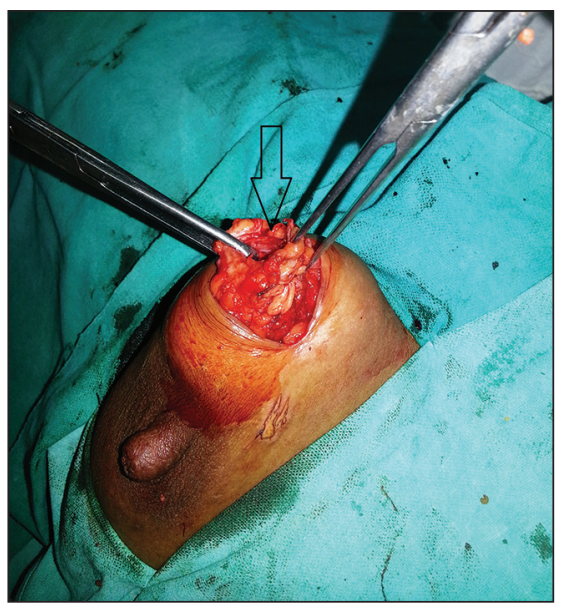

Figure 3: Per-operative photograph of the patient showing hydatid cyst of the breast

Preoperative chemotherapy using albendazole has been shown to decrease the incidence of recurrent disease. ${ }^{[9]}$ However, it may not prevent disease recurrence in a distant site. Recurrence is typically due to either incomplete cyst removal or previously unidentified cysts. The reported recurrence rates range from $2 \%$ to $25 \% \cdot{ }^{[10]}$ 


\section{CONCLUSION}

Primary hydatid cyst of the breast is a very rare condition. Preoperative diagnosis is difficult due to the disease mimicking a variety of conditions in old and young women. Hence, it should also be kept in the differential diagnosis of breast lump and proper evaluation should be performed preoperatively to rule out the same.

\section{REFERENCES}

1. Cancelo MJ, Martin M, Mendoza N. Preoperative diagnosis of a breast hydatid cyst using fine-needle aspiration cytology: A case report and review of the literature. J Med Case Rep 2012;6:293

2. Kosecik M, Karaoglanoglu M, Yamak B. Pericardial hydatid cyst presenting with cardiac tamponade. Can J Cardiol 2006;22:145-7.

3. Alamer A, Aldhilan A, Makanjuola D, Alkushi A. Preoperative diagnosis of hydatid cyst of the breast: A case report. Pan Afr Med J 2013;14:99.
4. Tutar N, Cakir B, Geyik E, Tarhan NC, Niron EA. Hydatid cysts in breast: Mammography and ultrasound findings. $\mathrm{Br}$ J Radiol 2006;79:e114-6.

5. Afroz N, Chaurasia JK, Maheshwari V, Singh G. Hydatid cyst: Unusual presentation as 'breast lump'. BMJ Case Rep 2014;2014. pii: bcr2014204273. doi: 10.1136/bcr-2014-204273.

6. Masroor I, Azeemuddin M, Khan S, Barakzai A. Hydatid disease of the breast. Singapore Med J 2010;51:e72-5.

7. Kamali NI, Raza MH, Rab AZ, Akhtar S. Isolated hydatid cyst of the breast-A case report. Indian J Surg 2013;75(Suppl 1):150-1.

8. Acar T, Gomcel Y, Guzel K, Yazgan A, Aydyn R. Isolated hydatid cyst of the breast. Scott Med J 2003;48:52-3.

9. Shams-Ul-Bari, Arif SH, Malik AA, Khaja AR, Dass TA, Naikoo ZA. Role of albendazole in the management of hydatid cyst liver. Saudi J Gastroenterol 2011;17:343-7.

10. Nunnari G, Pinzone MR, Gruttadauria S, Celesia BM, Madeddu G, Malaguarnera G. Hepatic echinococcosis: Clinical and therapeutic aspects. World J Gastroenterol 2012;18:1448-58.

How to cite this article: Siddiqui $\mathrm{B}$, et al. Primary hydatid disease of the breast clinically masquerading as a galactocele: A case report. J TransI Intern Med 2015;3:82-84

Source of Support: NIL, Conflict of Interest: NIL 